\title{
Resolved spectral variations of the centimetre-wavelength continuum from the $\rho$ Oph W photodissociation region
}

\author{
Simon Casassus ${ }^{\circledR}, 1 \star$ Matías Vidal $^{\circledR}, 2$ Carla Arce-Tord ${ }^{\circledR},{ }^{1}$ Clive Dickinson ${ }^{\circledR},{ }^{3,4}$ Glenn J. White, ${ }^{5,6}$ \\ Michael Burton ${ }^{\circledR},{ }^{7}$ Balthasar Indermuehle ${ }^{8}$ and Brandon Hensley ${ }^{9} \dagger$ \\ ${ }^{1}$ Departamento de Astronomía, Universidad de Chile, Camino El Observatorio 1515, Las Condes, Santiago, Chile \\ ${ }^{2}$ Facultad de Ingeniería, Núcleo de Astroquímica \& Astrofísica, Universidad Autónoma de Chile, Av. Pedro de Valdivia 425, Providencia, Santiago, Chile \\ ${ }^{3}$ Jodrell Bank Centre for Astrophysics, Alan Turing Building, Department of Physics and Astronomy, School of Natural Sciences, The University of \\ Manchester, Oxford Road, Manchester M13 9PL, UK \\ ${ }^{4}$ Cahill Center for Astronomy and Astrophysics, California Institute of Technology, Pasadena, CA 91125, USA \\ ${ }^{5}$ RAL Space, Rutherford Appleton Laboratory, Chilton, Didcot, Oxfordshire OX11 OQX, UK \\ ${ }^{6}$ Department of Physics and Astronomy, The Open University, Walton Hall, Milton Keynes MK7 6AA, England \\ ${ }^{7}$ School of Physics, University of New South Wales, Sydney NSW 2052, Australia \\ ${ }^{8}$ CSIRO Astronomy and Space Science, Marsfield, NSW 2122, Australia \\ ${ }^{9}$ Department of Astrophysical Sciences, Princeton University, Princeton, NJ 08544, USA
}

Accepted 2020 December 23. Received 2020 December 23; in original form 2020 October 1

\begin{abstract}
Centimetre-wavelength radio continuum emission in excess of free-free, synchrotron, and Rayleigh-Jeans dust emission (excess microwave emission, EME), and often called 'anomalous microwave emission', is bright in molecular cloud regions exposed to UV radiation, i.e. in photodissociation regions (PDRs). The EME correlates with infrared (IR) dust emission on degree angular scales. Resolved observations of well-studied PDRs are needed to compare the spectral variations of the cm-continuum with tracers of physical conditions and of the dust grain population. The EME is particularly bright in the regions of the $\rho$ Ophiuchi molecular cloud ( $\rho \mathrm{Oph}$ ) that surround the earliest type star in the complex, HD 147889, where the peak signal stems from the filament known as the $\rho$ Oph W PDR. Here, we report on Australia Telescope Compact Array observations of $\rho$ Oph W that resolve the width of the filament. We recover extended emission using a variant of non-parametric image synthesis performed in the sky plane. The multifrequency $17-39 \mathrm{GHz}$ mosaics reveal spectral variations in the centimetre-wavelength continuum. At $\sim 30$ arcsec resolutions, the $17-20 \mathrm{GHz}$ intensities tightly follow the mid-IR, $I_{\mathrm{cm}} \propto I(8 \mu \mathrm{m})$, despite the breakdown of this correlation on larger scales. However, while the $33-39 \mathrm{GHz}$ filament is parallel to Infrared Array Camera $8 \mu \mathrm{m}$, it is offset by 15-20 arcsec towards the UV source. Such morphological differences in frequency reflect spectral variations, which we quantify spectroscopically as a sharp and steepening high-frequency cutoff, interpreted in terms of the spinning dust emission mechanism as a minimum grain size $a_{\text {cutoff }} \sim 6 \pm 1 \AA$ that increases deeper into the PDR.
\end{abstract}

Key words: radiation mechanisms: general-ISM: clouds-ISM: individual objects: $\rho$ Oph, Rho Oph W filament, SR4, DoAr21 - photodissociation region - radio continuum: general - submillimetre: ISM.

\section{INTRODUCTION}

Cosmic microwave background anisotropy experiments have identified an anomalous diffuse foreground in the range of $10-90 \mathrm{GHz}$ (Kogut et al. 1996; Leitch et al. 1997), which was confirmed, in particular, by the WMAP (e.g. Gold et al. 2011) and Planck missions (e.g. Planck Collaboration 2016a). As summarized in Dickinson et al. (2018), this diffuse emission is correlated with the far-infrared (IR) thermal emission from dust grains on large angular scales, and at high galactic latitudes. The spectral index in specific intensity $\left(I_{v} \propto\right.$ $\nu^{\alpha}$ ) of the anomalous Galactic foreground is $\alpha_{\text {radio/IR }} \sim 0$ in the range $15-30 \mathrm{GHz}$ (Kogut et al. 1996), but any semblance to optically thin free-free is dissipated by a drop between 20 and $40 \mathrm{GHz}$, with

\footnotetext{
^E-mail: simon@das.uchile.cl

$\dagger$ Spitzer Fellow.
}

$\alpha_{\text {radio/IR }} \sim-0.85$ for high-latitude cirrus (Davies et al. 2006). The observed absence of $\mathrm{H} \alpha$ emission concomitant to radio free-free emission would require an electron temperature $T_{\mathrm{e}} \geq 10^{6} \mathrm{~K}$ to quench H I recombination lines (Leitch et al. 1997).

The past couple of decades have seen the detection of a dozen well-studied molecular clouds with bright centimetre-wavelength radiation in excess of the expected levels for free-free, synchrotron, or Rayleigh-Jeans dust emission alone (e.g. Finkbeiner et al. 2002; Watson et al. 2005; Casassus et al. 2006; Scaife et al. 2009; Scaife et al. 2010; Castellanos et al. 2011; Vidal et al. 2011; Tibbs et al. 2012; Cepeda-Arroita et al. 2020; Vidal et al. 2020). A common feature of all $\mathrm{cm}$-bright clouds is that they host conspicuous photodissociation regions (PDRs). The Planck mission has also picked up spectral variations in this excess microwave emission (EME) from source to source along the Gould belt where the peak frequency is $v_{\text {peak }} \sim$ 26-30 GHz, while $v_{\text {peak }} \sim 25 \mathrm{GHz}$ in the diffuse interstellar medium (ISM; Planck Collaboration 2013, 2016b). The prevailing interpre- 
Table 1. Log of observations for ATCA project C1845.

\begin{tabular}{lcccc}
\hline Date & Array $^{a}$ & Frequency $_{1}^{b}(\text { beam })^{d}$ & Frequency $_{2}^{c}(\text { beam })^{d}$ & Mosaic $^{e}$ \\
\hline 11-May-2009 & H 168 & $17481(33.8 \times 25.9 / 85)$ & $20160^{c}(28.6 \times 21.4 / 271)$ & 6 \\
12-May-2009 & H 168 & $5500(46.1 \times 34.5 / 278)$ & $8800(28.3 \times 19.2 / 83)$ & 1 \\
08-Jul-2009 & H 75 & $33157(17.2 \times 14.2 / 271)$ & $39157(13.9 \times 11.3 / 276)$ & 6 \\
\hline
\end{tabular}

${ }^{a}$ ATCA array configuration. Antenna CA06, stationed on the North spur at $\sim 4 \mathrm{~km}$ from the other five antennas in compact configuration, was not included in the analysis

${ }^{b, c}$ Centre frequencies for the two CABB IFs. Each IF is made up of $2048 \times 1 \mathrm{MHz}$ channels

${ }^{d}$ Natural-weights beam in arcsec, in the form $(B M A J \times B M I N / B P A)$, where BMAJ and BMIN are the full-width major and minor axis, and BPA is the beam PA in degrees East of North.

${ }^{e}$ Number of fields in $\rho$ Oph W.

tation for EME, also called anomalous microwave emission (AME), is electric-dipole radiation from spinning very small grains (VSGs), or 'spinning dust' (Draine \& Lazarian 1998a). A comprehensive review of all-sky surveys and targeted observations supports this spinning dust interpretation (Dickinson et al. 2018). The carriers of spinning dust remain to be identified, however, and could be polycyclic aromatic hydrocarbons (PAHs; e.g. Ali-Haïmoud 2014), nano-silicates (Hoang, Vinh \& Quynh Lan 2016; Hensley \& Draine 2017), or spinning magnetic dipoles (Hoang \& Lazarian 2016; Hensley \& Draine 2017). A contribution to EME from the thermal emission of magnetic dust (e.g. Draine \& Lazarian 1999) may be important in some regions (Draine \& Hensley 2012).

Increasingly refined models of spinning dust emission reach similar predictions for given dust parameters and local physical conditions (e.g. Ali-Haïmoud, Hirata \& Dickinson 2009; Hoang, Draine \& Lazarian 2010; Ysard \& Verstraete 2010; Silsbee, AliHaïmoud \& Hirata 2011). In addition, thermochemical PDR models estimate the local physical conditions that result from the transport of ultraviolet (UV) radiation (e.g. Le Petit et al. 2006). Therefore, observations of the centimetre-wavelength continuum in PDRs can potentially calibrate the spinning dust models and identify the dust carriers. The radio continua from PDRs may eventually provide constraints on physical conditions.

In particular, $\rho$ Ophiuchi $(\rho \mathrm{Oph}) \mathrm{W}$, the region of the $\rho$ Oph molecular cloud exposed to UV radiation from HD 147889, is amongst the closest examples of PDR, lying at a distance of 138.9 pc (Gaia Collaboration 2018). It is seen edge-on and extends over $\sim 10 \times 3$ arcmin. $\rho$ Oph is a region of intermediate-mass star formation (Pattle et al. 2015; White et al. 2015). It does not host a conspicuous H II region, by contrast to the Orion Bar, another wellstudied PDR, where UV fields are $\sim 100$ times stronger. $\rho$ Oph W has been extensively studied in the far-IR atomic lines observed by ISO (Liseau et al. 1999; Habart et al. 2003). Observations of the bulk molecular gas in $\rho$ Oph, through ${ }^{12} \mathrm{CO}(1-0)$ and ${ }^{13} \mathrm{CO}(1-0)$, are available from the COMPLETE data base (Ridge et al. 2006). While the HD 147889 binary has the earliest spectral types in the complex (B2IV and B3IV Casassus et al. 2008), the region also hosts two other early-type stars: S 1 (which is a close binary including a B4V star, Lada \& Wilking 1984), and SR 3 (with spectral type B6V, Elias 1978). Both S 1 and SR 3 are embedded in the molecular cloud and are surrounded by bright IR nebulosity, very conspicuous in the SpitzerInfrared Array Camera (IRAC) $8 \mu \mathrm{m}$ map (as provided by the $c 2 d$ Spitzer Legacy Survey). An image of the region including the relative positions of these three early-type stars can be found in Casassus et al. (2008, their fig. 4) or in Arce-Tord et al. (2020, their fig. 2).

Cosmic Background Imager (CBI) observations showed that the surprisingly bright centimetre-wavelength continuum from $\rho$ Oph, with a total WMAP $33 \mathrm{GHz}$ flux density of $\sim 20 \mathrm{Jy}$, peaks in $\rho$ Oph W (Casassus et al. 2008). The WMAP spectral energy distribution
(SED) is fit by spinning dust models (within 45 arcmin, Casassus et al. 2008), as well as the Planck SED (within 60 arcmin; Planck Collaboration 2011). However, the peak at all IR wavelengths, i.e. the circumstellar nebula around S 1 , is undetectable in the CBI data. Upper limits on the $\mathrm{S} 1$ radio flux density and correlation tests with the IRAC $8 \mu \mathrm{m}$ map rule out a linear radio/IR relationship within the CBI 45 arcmin primary beam (which encompasses the bulk of $\rho$ Oph by mass). This breakdown of the radio-IR correlation in the $\rho$ Oph complex is further pronounced at finer angular resolutions, with observations from the CBI 2 upgrade to CBI (Arce-Tord et al. 2020).

Thus, while the centimetre-wavelength and near- to mid-IR signals in the $\rho \mathrm{OphW}$ filament correlate tightly, as expected for EME, this correlation breaks down in the $\rho$ Oph complex as a whole, when including also the circumstellar nebula around S 1 . Under the spinning dust hypothesis, this breakdown points at environmental factors that strongly impact the spinning dust emissivity per nucleon. Dust emissivities in the near-IR, from the stochastic heating of VSGs, are roughly proportional to the energy density of UV radiation: $I_{\mathrm{IR}} \propto$ $G_{\circ} N_{\mathrm{VSG}}$. For a universally constant spinning dust emissivity $\frac{j_{\mathrm{lcm}}}{n_{\mathrm{H}}}$, we expect $I_{\mathrm{cm}} \propto N_{\mathrm{VSG}} \propto I_{\mathrm{IR}} / G_{\circ}$. This correlation is marginally ruled out in the CBI data, which thus point at emissivity variations within the source (Casassus et al. 2008). The CBI 2 observations, with a finer beam, reveal that $\frac{j_{1 \mathrm{~cm}}}{n_{\mathrm{H}}}$ varies by a factor of at least 26 at $3 \sigma$ (Arce-Tord et al. 2020).

Here, we present observations of $\rho$ Oph W acquired with the Australia Telescope Compact Array (ATCA), with the aim of resolving the structure of this PDR on $\sim 30$ arcsec scales. The structure of this article is as follows: Section 2 describes the ATCA observations, Section 3 analyses the spectral variations in these multifrequency data, Section 4 reports limits on the Carbon radio-recombination lines, which trace the ions possibly responsible for the grain spin-up, and Section 5 concludes. Technical details on image reconstruction are given in the Appendix.

\section{OBSERVATIONS}

\subsection{Calibration and imaging}

We covered the central region of $\rho \mathrm{Oph} \mathrm{W}$ with Nyquist-sampled mosaics, as detailed in the log of observations (see Table 1). The primary calibrator was J1934-638 (for flux and bandpass), and the secondary calibrator was J1622-253 (for phase). The Compact Array Broadband Backend (CABB; Wilson et al. 2011) provided a total of $4 \mathrm{GHz}$ bandwidth split into two IFs. The data were calibrated using the MIRIAD package (Sault, Teuben \& Wright 1995) and following the standard procedure for ATCA.

Traditional image synthesis techniques based on the Clean algorithm (Högbom 1974) are best suited for compact sources. Our 

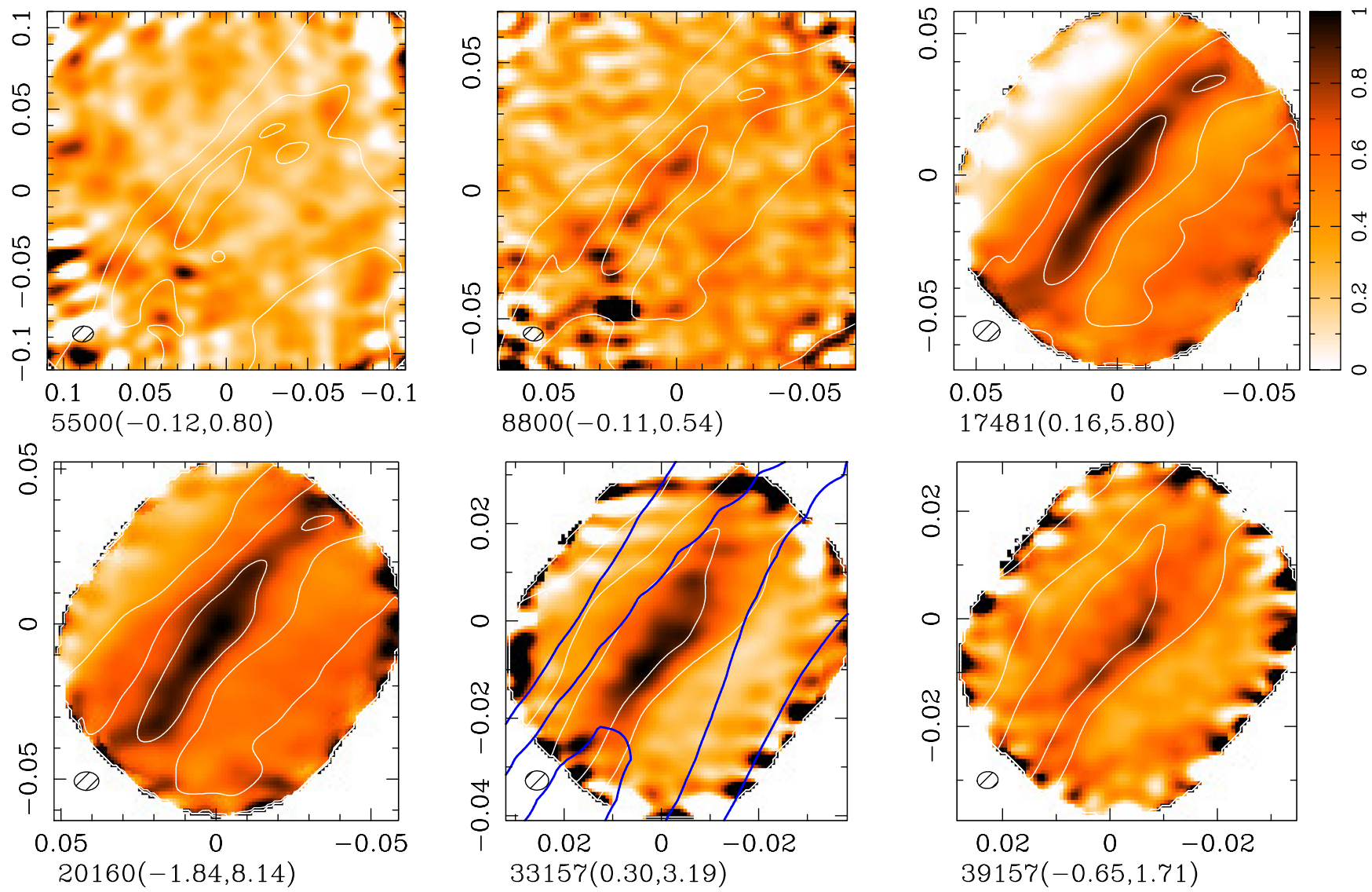

Figure 1. ATCA observations of $\rho$ Oph $\mathrm{W}$, and comparison with the $8 \mu \mathrm{m}$ emission, illustrating the systematic shift of the filament towards HD 147889 (to the south-west) with increasing frequency. $x$ - and $y$-axis show offset RA and DEC from $\rho$ Oph W $\left(\mathrm{J} 200016^{\mathrm{h}} 25^{\mathrm{m}} 57^{\mathrm{s}}\right.$ to $\left.24^{\mathrm{d}} 20^{\mathrm{m}} 50^{\mathrm{s}}\right)$, in degrees of arc. Note that fields of view are different as appropriate for each frequency. The restored mosaics are shown in colour scale. Identical restorations of IRAC $8 \mu \mathrm{m}$ visibilities, obtained by simulating the same ATCA observations at each frequency, are shown in white contours, with levels at $0.25,0.5$, and 0.75 times the peak IRAC $8 \mu \mathrm{m}$ restored intensities. All colour scales range linearly from 0 to 1 , and all images have been linearly scaled and normalized so that their entire intensity range, shown in parenthesis at the bottom of each image, is matched by the colour scale. Frequencies are given in $\mathrm{MHz}$ at the bottom left of each image. At $33.157 \mathrm{GHz}$, we have also overlaid the CBI2 UVMEM image of the $31 \mathrm{GHz}$ continuum from Arce-Tord et al. (2020), in blue contours at 0.5, 0.75, and 0.95 times the peak.

initial trials with Miriad and Clean did not recover much signal from the ATCA observations of $\rho$ Oph W, where the signal fills the primary beam, and poor $u v$-coverage resulted in strong negative sidelobes. We thus designed a special purpose image synthesis algorithm, which we call SKYMEM, based on non-parametric model images rather than on the collection of delta-functions used by Clean. Full details on image reconstruction are given in Appendix A. SKYMEM yields restored images defined in a similar way as for Clean.

Probably the most important feature of SKYMEM, which allowed the recovery of the missing spatial frequencies, is the use of an image template as initial condition. For adequate results, this template must tightly correlate with the signal, and in this application we used the IRAC $8 \mu \mathrm{m}$ mosaic of the entire $\rho$ Oph region, which has an angular resolution of 2 arcsec. The centimetre-wavelength radio signal in the $\rho$ Oph W filament is known to correlate with IRAC $8 \mu \mathrm{m}$ (Casassus et al. 2008; Arce-Tord et al. 2020). Since its resolution is much finer than that of the ATCA signal we aim to image, and as it is relatively less crowded by point sources compared to the shorter wavelengths, we adopted the IRAC $8 \mu \mathrm{m}$ mosaic as the SKYMEM template after point-source subtraction by median filtering. This template is the same as that used in (Casassus et al. 2008), and is also shown here in the Appendix.
The SKYMEM mosaic for the ATCA data in $\rho \mathrm{Oph} \mathrm{W}$ is shown in Fig. 1. We can readily identify morphological variations with frequency, so that the radio filament appears to systematically shift towards the south-west, i.e. towards HD 147889, with increasing frequency. There are, however, other frequency dependent variations which may be due to interferometer filtering in different $u v$ -coverages. Since the morphology of the centimetre-wavelength filament is similar to IRAC $8 \mu \mathrm{m}$, we compare the ATCA mosaics at each frequency with SKYMEM reconstructions of IRAC $8 \mu \mathrm{m}$ after filtering for the corresponding $u v$-coverage using MIRIAD task UVMODEL. This accounts for the spatial filtering by the interferometer and allows a robust comparison between wavebands.

\subsection{Relevant point sources in the ATCA mosaics}

The ATCA angular resolutions allow to separate point sources from the diffuse signal in the filament, such as proto-planetary discs whose steeply rising thermal continuum emission may be relevant at the higher frequencies. In particular, the disc around SR 4, at J2000 16:25:56 to 24:20:48.2 and near the centre of coordinates in Fig. 1, probably corresponds to the peak signal at $39 \mathrm{GHz}$. It is best seen in the Clean map at $39 \mathrm{GHz}$ shown in Fig. A1, since the extended 
Table 2. ATCA - IRAC $8 \mu \mathrm{m}$ correlation statistics.

\begin{tabular}{lll}
\hline Frequency $^{a}$ & $a \pm \sigma(a)^{b}$ & $r_{w}{ }^{c}$ \\
\hline 8800 & $0.13 \pm 0.01$ & 0.52 \\
17481 & $1.63 \pm 0.01$ & 0.89 \\
20160 & $3.05 \pm 0.02$ & 0.95 \\
33157 & $2.77 \pm 0.05$ & 0.83 \\
39157 & $1.38 \pm 0.04$ & 0.61 \\
\hline
\end{tabular}

${ }^{a}$ Centre frequency in $\mathrm{MHz} .{ }^{b}$ Dimensionless correlation slope $I_{\mathrm{ATCA}}=$ $a I_{\mathrm{IRAC}} 8 \mu \mathrm{m} \cdot{ }^{c}$ Linear-correlation coefficient.

signal is filtered in this Clean image, and we can infer a flux density of $0.34 \pm 0.03 \mathrm{mJy}$. This point source has been subtracted from the $39 \mathrm{GHz}$ data shown in Fig. 1 and in the subsequent analysis.

A different point source dominates the signal in the 5 and $8 \mathrm{GHz}$ maps. This source coincides with the DoAr 21 variable star, which is surrounded by near-IR nebulosity and filaments (Garufi et al. 2020) but is not detected in the Atacama Large Millimetre Array (ALMA) continuum at $230 \mathrm{GHz}$ (Cieza et al. 2019). In these ATCA data, its flux density is $0.9 \pm 0.1 \mathrm{mJy}$ at $5 \mathrm{GHz}$ and $4.2 \pm 0.05 \mathrm{mJy}$ at $8 \mathrm{GHz}$. This point source is also picked up in Clean images of the 17 and $20 \mathrm{GHz}$ ATCA data that include the long baselines that join the five antennas in the compact configuration with antenna CA06, stationed at $\sim 4.4 \mathrm{~km}$ on the North spur of the ATCA array. In these data the nebular emission is entirely filtered out, and only DoAr 21 remains, with flux densities of $0.6 \pm 0.03 \mathrm{mJy}$ at $17 \mathrm{GHz}$ and $0.2 \pm 0.04 \mathrm{mJy}$ at $20 \mathrm{GHz}$. Antenna CA06 is not included in the analysis of the nebular signal. DoAr 21 is not subtracted in the analysis as it is located outside the field of the higher frequencies, and its flux is negligible compared to the nebular emission at 17 and $20 \mathrm{GHz}$.

\subsection{ATCA - IRAC $8 \mu \mathrm{m}$ cross-correlations}

The signal in the ATCA reconstructions of $\rho$ Oph W follows quite tightly the IRAC filament, as also seen in other observations at similar angular resolutions (e.g. in LDN 1246, observed at 25 arcsec by Scaife et al. 2010, using the Arcminute Microkelvin Imager). Table 2 lists cross-correlation slopes and statistics. The slopes $a(v)$ are cal- culated on the SKYMEM-restored mosaics, with $I^{R}(v)=a(v) I_{8 \mu \mathrm{m}}$,

$a(v)=\frac{\sum_{i} I_{8 \mu \mathrm{m}}\left(\nu, \vec{x}_{i}\right) I^{R}\left(\nu, \vec{x}_{i}\right) w_{R}\left(\nu, \vec{x}_{i}\right)}{\sum_{i}\left[I_{8 \mu \mathrm{m}}\left(\nu, \vec{x}_{i}\right)\right]^{2} w_{R}\left(\nu, \vec{x}_{i}\right)}$,

where the weight image $w_{R}(v)=1 / \sigma_{R}(v)^{2}$, and $\sigma_{R}(v)$ is calculated with a linear mosaic of MIRIAD's sensitivity maps for each pointing (see Appendix A, equation A9). The IRAC $8 \mu \mathrm{m}$ comparison images, $I_{8 \mu \mathrm{m}}\left(v, \vec{x}_{i}\right)$, have been filtered for the frequency-dependent $u v$ coverage, and scaled in intensity to approximate the range of intensities observed in EME sources (as described in Appendix A). Specifically, we scale the IRAC $8 \mu \mathrm{m}$ mosaic by the slope of the CBI/IRAC $8 \mu \mathrm{m}$ correlation measured in M 78 by Castellanos et al. (2011). For instance, the ratio of centimetre-wavelength specific intensities relative to IRAC $8 \mu \mathrm{m}$ are typically 3.05 times higher in $\rho$ Oph W at $20 \mathrm{GHz}$ than in M 78 at $31 \mathrm{GHz}$. The slopes $a(v)$ are therefore dimensionless, and can be used as an SED indicator, albeit in arbitrary units.

The linear-correlation coefficient $r_{w}$ in Table 2 corresponds to

$r_{w}=\frac{\sum_{i}\left(x_{i}-x_{\circ}\right)\left(y_{i}-y_{\circ}\right)}{\sqrt{\sum_{i}\left(x_{i}-x_{\circ}\right)^{2} \sum_{i}\left(y_{i}-y_{\circ}\right)^{2}}}$,

where $x_{\circ}=\frac{\sum_{i}\left(x_{i} w_{i}\right)}{\sum_{i} w_{i}}, y_{\circ}=\frac{\sum_{i}\left(y_{i} w_{i}\right)}{\sum_{i} w_{i}}$, and $x_{i}=I_{8 \mu \mathrm{m}}\left(\nu, \vec{x}_{i}\right), y_{i}=$ $I_{\mathrm{ATCA}}\left(\nu, \vec{x}_{i}\right)$. According to this correlation test, the best match to IRAC $8 \mu \mathrm{m}$ corresponds to $20 \mathrm{GHz}$.

\section{SPECTRAL VARIATIONS}

\subsection{Morphological trends with frequency}

The morphological variations with frequency apparent in Fig. 1 can also be measured with intensity profiles across the $\rho$ Oph W filament. The intensity profiles shown in Fig. 2 were generated by extracting 2 arcmin-wide cuts orthogonal to the filament. The images were rotated so that their $y$-axis is aligned at a position angle of $-40 \mathrm{deg}$ East of North, and roughly coincident with the direction of the filament. One-dimensional profiles were then obtained by averaging the $2 \mathrm{D}$ specific intensities along the $y$-axis.

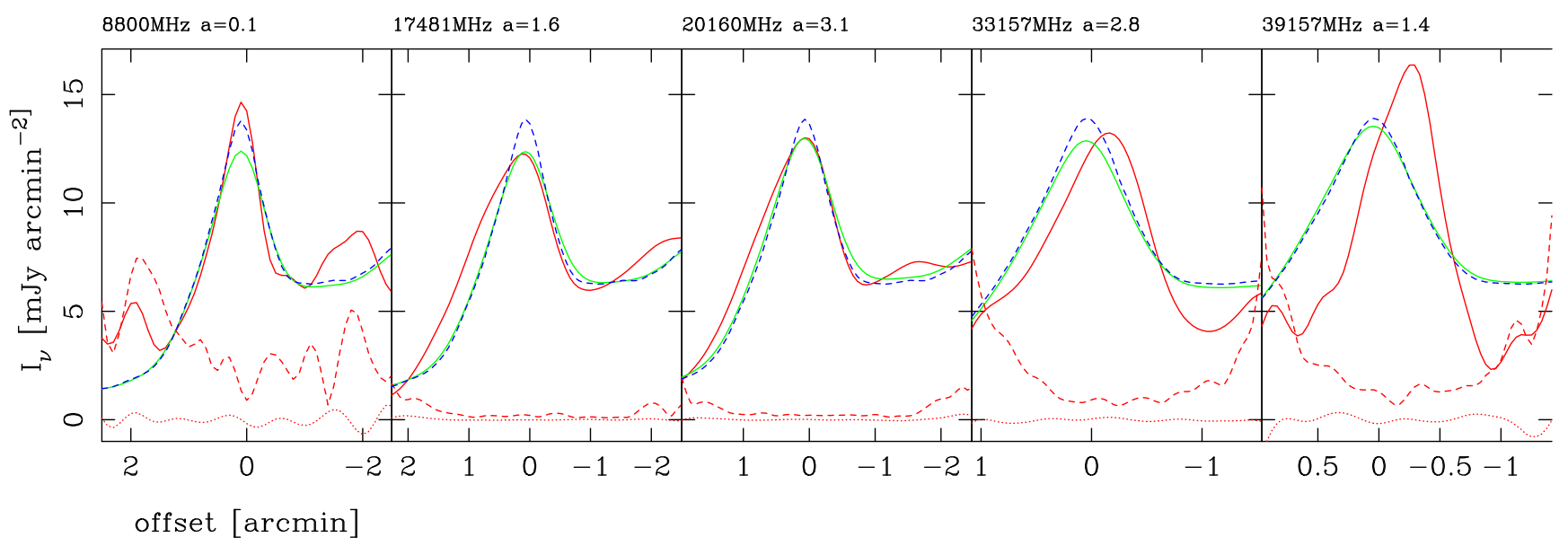

Figure 2. Profiles from the restored ATCA mosaics extracted perpendicularly to the $\rho$ Oph W PDR. Frequencies are indicated on the top left. The following number is the radio-IR correlation slope $a$ (same as in Table 2 and described in Section 2.3). The profile at each frequency is divided by $a$. The $x$-axis shows offset in arcmin from the reference position, at J2000 16:25:57.984 to 24:20:37.760. $y$-axis shows specific intensities averaged in a region \pm 1 arcmin along the filament. The ATCA profiles are shown in red, with the restored image in solid line, its average residuals in dotted line, and the rms-dispersion of residuals in dashed line. The blue dashed line is the average profile of the IRAC $8 \mu \mathrm{m}$ template, which is the original IRAC image filtered and scaled by a reference radio/IR correlation slop (see Section 2.3). The green dashed line is the corresponding profile of a simulation of the ATCA observations and SKYMEM restoration. 

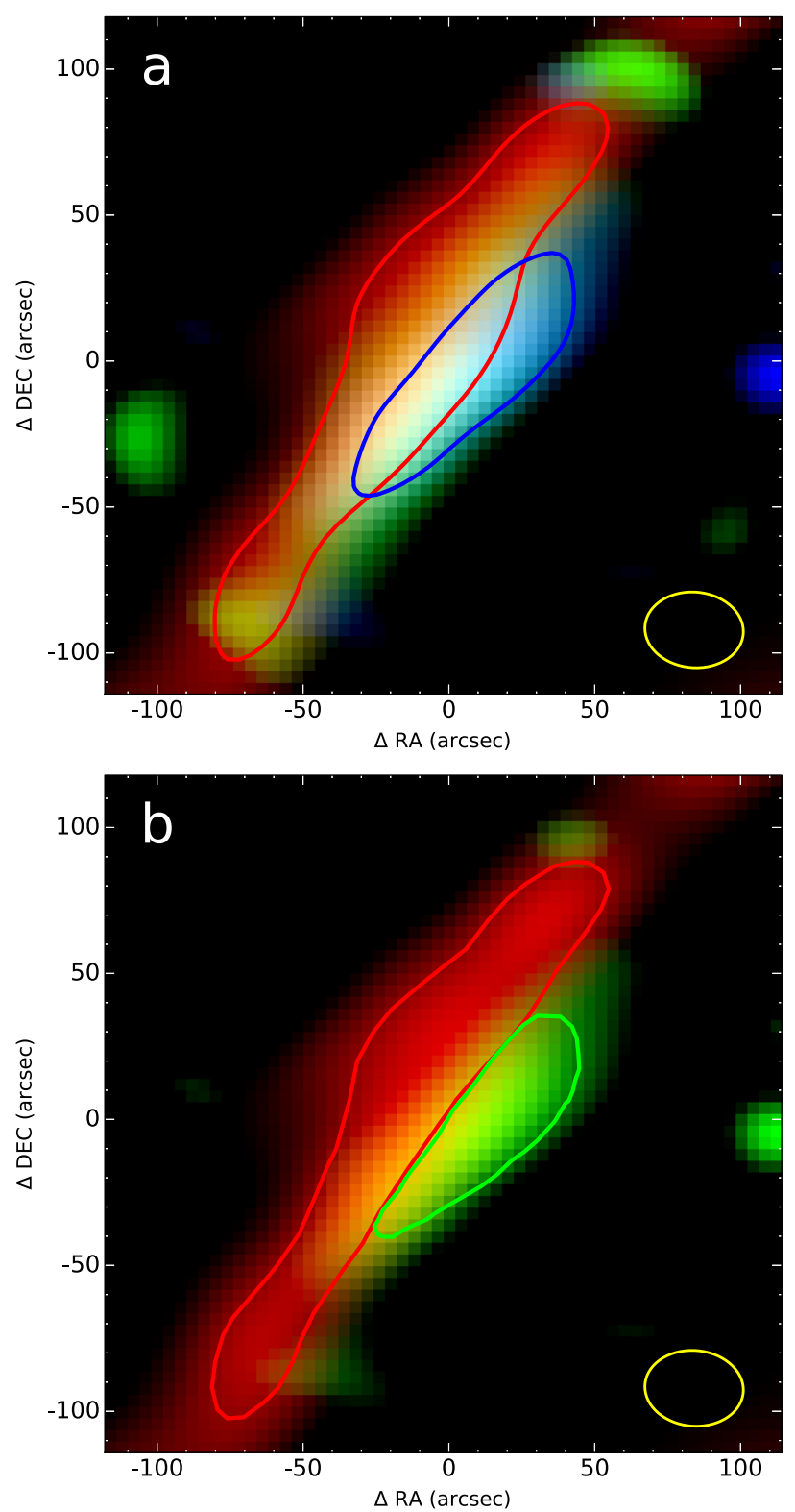

Figure 3. The $\rho \mathrm{Oph} \mathrm{W}$ filament shifts towards the exciting star with increasing frequency, as illustrated in these colour-coded versions of the restored maps shown in Fig. 1, after subtraction of the SR 4 point source at $39 \mathrm{GHz}$, and degraded to a common angular resolution. (a): This RGB image is linearly scaled to cover the whole range of intensities at each frequency: $17 \mathrm{GHz}$ in red and $33 \mathrm{GHz}$ in green and $39 \mathrm{GHz}$ in blue. The contours are taken at $85 \%$ peak level at $17 \mathrm{GHz}$ and $80 \%$ peak level at $39 \mathrm{GHz}$, and are drawn with matching colours. The common beam corresponds to that of the $17 \mathrm{GHz}$ map, and is indicated by the yellow ellipse (see Table 1). (b) In this red-green version, with $17 \mathrm{GHz}$ in red and $39 \mathrm{GHz}$ in green, we illustrate the photometric apertures, or masks, used to measure the SED (Section 3.2). The contour levels are the same as in a), with $17 \mathrm{GHz}$ in red and $39 \mathrm{GHz}$ in green, but are modified to avoid overlap.

A dual-frequency comparison of the maps degraded to the same angular resolution is shown in Fig. 3. The common beam is that of the $17 \mathrm{GHz}$ measurements (see Table 1). The contours in Fig. 3(b) compare the morphologies at 17 and $39 \mathrm{GHz}$ and correspond to the two photometric apertures used for the extraction of the SED, they are thus edited from genuine contour levels to avoid overlap. The
Table 3. ATCA mean intensities and their ratio measured in the two masks shown in Fig. 3. The quoted uncertainties refer to the thermal errors only the actual uncertainties should include 10 per cent in quadrature, and have been applied to the ratio $\mathrm{I}_{v}^{39} / \mathrm{I}_{v}^{17}$.

\begin{tabular}{lccc}
\hline $\begin{array}{l}\text { Frequency } \\
(\mathrm{GHz})\end{array}$ & $\begin{array}{c}17 \mathrm{GHz} \text { mask } \\
\left(\times 10^{4} \mathrm{Jy} \mathrm{sr}^{-1}\right)\end{array}$ & $\begin{array}{c}39 \mathrm{GHz} \text { mask } \\
\left(\times 10^{4} \mathrm{Jy} \mathrm{sr}^{-1}\right)\end{array}$ & $\begin{array}{c}\text { Ratio } \\
I_{v}^{39} / I_{v}^{17}\end{array}$ \\
\hline 5.5 & $<0.3^{a}$ & $<0.3^{a}$ & - \\
8.8 & $1.83,0.04$ & $1.69,0.07$ & $0.9,0.1$ \\
17.5 & $22.93,0.03$ & $20.04,0.04$ & $0.9,0.1$ \\
20.2 & $43.79,0.06$ & $39.99,0.08$ & $0.9,0.1$ \\
33.2 & $37.32,0.13$ & $40.52,0.19$ & $1.1,0.1$ \\
39.2 & $18.05,0.08$ & $23.99,0.11$ & $1.3,0.2$ \\
\hline
\end{tabular}

${ }^{a} 3 \sigma$ upper limits using the dispersion of residuals.

contours also illustrate the westward shift of the peak emission in frequency.

We conclude that the centimetre-wavelength signal from the $\rho$ Oph W filament shifts towards higher frequencies with decreasing distance to the exciting star HD 147889. In other words, these morphological trends with frequency point at spectral variations in the EME spectrum when emerging from the PDR towards the UV source. It is interesting to note that a similar spectral trend has been reported in the PDR surrounding the $\lambda$ Ori region, where the EME signal peaks at increasingly higher frequencies towards the UV source (Cepeda-Arroita et al. 2020). The next Section addresses how the spectral trends implicit in the morphological trends observed in $\rho$ Oph W could be related to varying physical conditions under the spinning dust hypothesis.

\subsection{Spectral energy distribution}

The multifrequency radio maps of $\rho$ Oph W allow for estimates of its SED between 5 and $39 \mathrm{GHz}$. The morphological trends in frequency should be reflected in variations of the SED between the emission originating around the $17 \mathrm{GHz}$ peak and the emission coming from the vicinity of the $39 \mathrm{GHz}$ peak. Such multifrequency analysis requires smoothing the data to a common beam, which is that of the coarsest observations, at $17 \mathrm{GHz}$. Once smoothed, we measured the mean intensity in each map inside the two masks shown in Fig. 3. Interferometer data are known to be affected by flux-loss, i.e. missing flux from large angular scales not sampled by the $u v$-coverage of the interferometer. By using a prior image not affected by flux-loss, i.e. as defined in Appendix A, the SKYMEM algorithm allows to recover such flux-loss under the assumption of linear correlation with the prior. Our simulations using the prior image recovered the missing flux exactly, but since the centimetrewavelength signal does not exactly follow the prior, biases in the flux-loss correction scheme may affect the SEDs reported here. We expect such biases to be small and include them in the absolute calibration error of 10 per cent, given the tight correlation with the near-IR tracers and especially with the IRAC $8 \mu \mathrm{m}$ image used to build the prior image.

Table 3 lists the mean intensities $\left\langle I_{v}\right\rangle$ measured within the 17 and $39 \mathrm{GHz}$ masks, $\mathcal{M}_{17}$ and $\mathcal{M}_{39}$, for the six frequencies that we observed. We weighted the photometric extraction using the noise image $\sigma_{R}(\vec{x})$ given in equation (A12), i.e.

$$
\left\langle I_{v}^{k}\right\rangle=\frac{\sum_{\vec{x}_{j} \in \mathcal{M}_{k}} w_{R}\left(\vec{x}_{j}\right) I_{v}\left(\vec{x}_{j}\right)}{\sum_{\vec{x}_{j} \in \mathcal{M}_{k}} w_{R}\left(\vec{x}_{j}\right)},
$$




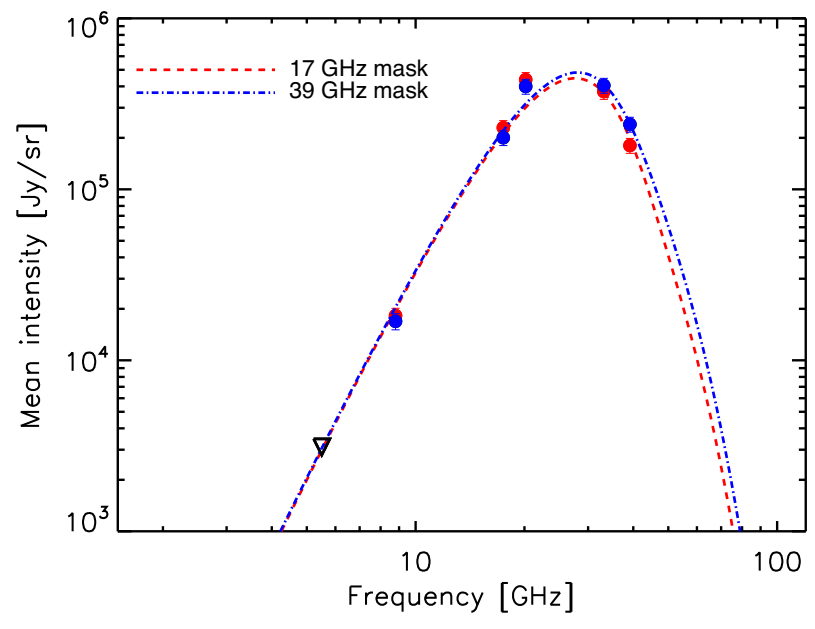

Figure 4. SED of the $\rho$ Oph W filament measured between 5.5 and $39 \mathrm{GHz}$ within the two apertures shown in Fig. 3. The red and blue lines correspond to the best-fitting spinning dust models.

for each mask $\mathcal{M}_{k}$, and with $w_{R}=1 / \sigma_{R}^{2}$. The associated error is

$\sigma\left(I_{v}^{k}\right)=\sqrt{\frac{N_{\text {beam }}}{\sum_{\vec{x}_{j} \in \mathcal{M}_{k}} w_{R}\left(\vec{x}_{j}\right)}}$,

where $N_{\text {beam }}$ is the number of pixels in a beam. The same SEDs are also plotted in Fig. 4, where we have included a conservative 10 per cent systematic uncertainty.

When including the 10 per cent absolute flux calibration uncertainty, the difference between the SEDs extracted in the two photometric apertures is not significant. The $\chi^{2}$ distribution yields that the two SEDs are different at 75 per cent confidence. Only the $39 \mathrm{GHz}$ average intensities appear to differ at the 95 per cent confidence level, or $2 \sigma$. Table 3 none the less lists the ratio between the measured intensities in each region $I_{v}^{39} / I_{v}^{17}$, as this ratio systematically increases with frequency, which may reflect the morphological trends. In Fig. 4, the spectra of the two regions show a steep drop after reaching the peak, at a frequency of $\sim 30 \mathrm{GHz}$. We can notice that the difference in measured intensity between the two regions is largest at $39 \mathrm{GHz}$. The emission from the $39 \mathrm{GHz}$ mask shows a spectrum brighter at higher frequencies than the emission coming from the $17 \mathrm{GHz}$ mask. This is interesting as the $39 \mathrm{GHz}$ mask is shifted towards the direction of the illuminating star HD 147889.

\subsubsection{SED modelling}

Previous works have shown that the $\mathrm{cm}$-wave emission from this region is dominated by EME and does not have major contributions from synchrotron or free-free emission, and that its SED on degree angular scales is adequately fit by spinning-dust models (see e.g. Casassus et al. 2008; Planck Collaboration 2011; Arce-Tord et al. 2020). Here, we ask the question of what are the consequences of the SED that we measure with ATCA, on arcminute scales, for the physical conditions and grain populations within the cloud, and under the spinning-dust hypothesis. Since the ATCA mosaic of $\rho$ Oph W is clear of any detectable free-free emission, as shown by the $5 \mathrm{GHz}$ map, we used only a spinning dust component, as calculated using the SPDUST code (Ali-Haïmoud et al. 2009).

The spinning dust emission depends on a large $(\sim 10)$ number of parameters that determine environmental properties: the gas density $\left(n_{\mathrm{H}}\right)$, the gas temperature $(\mathrm{T})$, the intensity of the radiation field

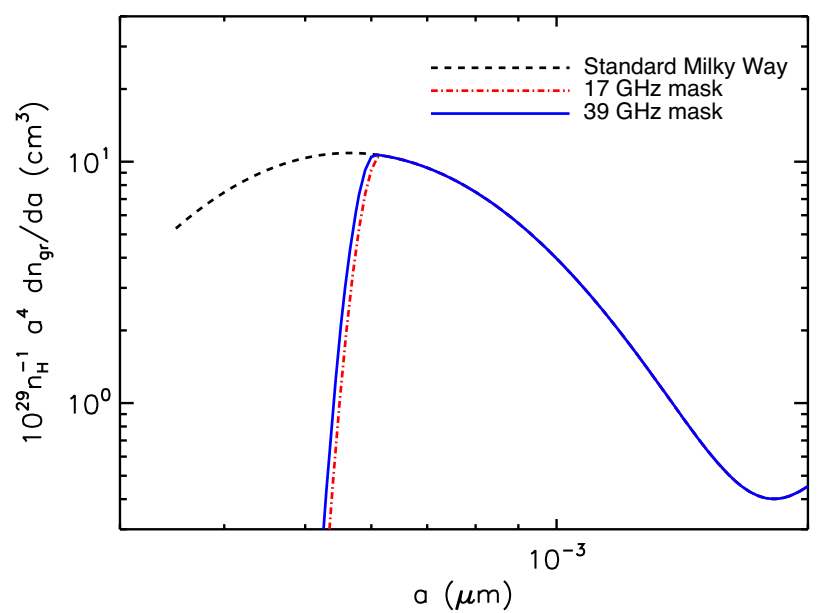

Figure 5. Grain size distribution for sizes around $1 \mathrm{~nm}$ for three cases. In black is the prescription from Weingartner \& Draine (2001) for typical Milky Way parameters. In red and blue are the distributions arising from the spinning dust fit in the two studied regions. In these cases, a cut-off is needed in order to fit the SEDs in Fig. 4.

(parametrized in terms of the starlight intensity relative to the average starlight background, $\chi$ ), the ionized hydrogen fractional abundance $x_{\mathrm{H}} \equiv n_{\mathrm{H}^{+}} / n_{\mathrm{H}}$, and the ionized carbon fractional abundance $x_{\mathrm{C}} \equiv$ $n_{\mathrm{C}^{+}} / n_{\mathrm{H}}$. In addition, the spinning dust emissivities also depend on the grain micro-physics, such as the grain-size distribution and the average dipole moment per atom for the dust grains. We will assume that the emission detected by ATCA is originated by spinning PAHs. The motivation for this assumption is the excellent correlation between the radio emission and the $8 \mu \mathrm{m}$ map in this region (Casassus et al. 2008; Arce-Tord et al. 2020).

In order to fit the ATCA data using the SPDUST code, we fixed some of the parameters that are well constrained in the literature for this region. Habart et al. (2003) modelled the mid-IR line emission using a PDR code and derived physical parameters for the $\rho$ Oph W filament. Using their results, we fixed the gas temperature and the intensity of the radiation field. For the ionized hydrogen and carbon abundances, we took the idealized values for PDRs that are listed in Draine \& Lazarian (1998b). We then fitted the SEDs using only three free parameters: gas density $\left(n_{\mathrm{H}}\right)$, average dipole $(\beta)$, and an additional parameter of the grain size distribution $\left(a_{\text {cutoff }}\right)$ that represents the minimum PAH size that is present in the region. This last parameter is necessary to avoid shifting the spinning dust peak to frequencies higher than $\sim 30 \mathrm{GHz}$, as predicted by spinning dust models for an ISM dust distribution in dense conditions such as in this PDR. We note that some of the parameters in SPDUST are expected to be highly correlated, for example the gas density, temperature, and radiation field. We avoid these degeneracies by fixing most of the parameters to the physical conditions already inferred for this region.

In SPDUST, the grain size distribution is parametrized as in Weingartner \& Draine (2001), where the contribution from PAHs is characterized by two lognormal distributions. A typical curve using standard parameters for the Milky Way is shown in black in Fig. 5. We introduced the $a_{\text {cutoff }}$ parameter in order to adjust the region of the grain size distribution that is most relevant to the spinning dust emission: the population of the smallest grains. This additional parameter, $a_{\text {cutoff }}$, corresponds to a characteristic size below which we apply an exponential cut-off modulating the size distribution, so effectively defining a minimum size for the PAHs. 
Table 4. SPDUST2 fit parameters. Parameters without uncertainty were fixed to the ones reported in Habart et al. (2003).

\begin{tabular}{|c|c|c|}
\hline Parameter & Mask $_{17 \mathrm{GHz}}$ & Mask $_{39 \mathrm{GHz}}$ \\
\hline$n_{\mathrm{H}}\left[10^{3} \mathrm{~cm}^{-3}\right]$ & \multicolumn{2}{|c|}{3.2} \\
\hline $\mathrm{T}[\mathrm{K}]$ & \multicolumn{2}{|c|}{300.0} \\
\hline$\chi$ & \multicolumn{2}{|c|}{400.0} \\
\hline$x_{\mathrm{H}} \equiv n_{\mathrm{H}^{+}} / n_{\mathrm{H}}(\mathrm{ppm})$ & \multicolumn{2}{|c|}{1200.0} \\
\hline$x_{\mathrm{C}} \equiv n_{\mathrm{C}^{+}} / n_{\mathrm{H}}(\mathrm{ppm})$ & \multicolumn{2}{|c|}{300.0} \\
\hline$y \equiv 2 n\left(\mathrm{H}_{2}\right) / \mathrm{n}_{\mathrm{H}}$ & \multicolumn{2}{|c|}{0.0} \\
\hline$\beta$ & \multicolumn{2}{|c|}{35.2} \\
\hline$a_{\text {cutoff }}(\AA)$ & $6.17 \pm 0.04$ & $6.07 \pm 0.04$ \\
\hline$\chi_{r}^{2}$ & 2.9 & 2.4 \\
\hline
\end{tabular}

The data in the SEDs were fitted using the IDL routine mp $\mathrm{i}$ t $f$ un (Markwardt 2009), which uses the Levenberg-Marquardt leastsquares fit to a function. We performed the fit using the SPDUST model for the two regions shown in Fig. 3. The result from this initial fit gave very similar values between the two regions for $n_{\mathrm{H}}(3.0 \pm 1.5$ versus $3.1 \pm 2.7)$ and $\beta(38.7 \pm 5.9$ versus $35.1 \pm 8.4)$. We thus

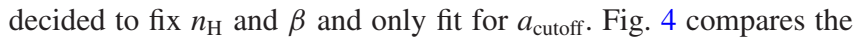
best-fitting SPDUST 2 model curves with the SED data points.

The result of our fits are summarized in Table 4 . The key parameter to account for the observed maximum at $30 \mathrm{GHz}$ is $a_{\text {cutoff }}$, without which the peak would shift towards $\sim 90 \mathrm{GHz}$ if fixing the physical conditions to those determined independently in this PDR by Habart et al. (2003). The difference in the free parameter, $a_{\text {cutoff }}$, between the two regions is only $1.8 \sigma$ but it seems to go in the direction expected in a PDR. The minimum grain size $a_{\text {cutoff }}$ is slightly larger in the fit of the $17 \mathrm{GHz}$ mask data. This means that in this region, there is a slightly lower abundance of the smallest grains, compared to the other region. This behaviour is in agreement with intuition, as the $39 \mathrm{GHz}$ mask is more exposed to the radiation from HD 147889 , which can result in a larger number of the smallest PAHs due to the fragmentation of larger ones. This provides a possible interpretation for the strong morphological differences with frequency, which is reflected in the local SEDs. Vidal et al. (2020) recently concluded that variations in the grain size distribution are also needed to explain spinning dust morphology in the translucent cloud LDN 1780.

The difference in $a_{\text {cutoff }}$ between the two regions used to link the multifrequency morphological variations with spectral trends may seem small. However, it is consistent with equipartition of rotational energy (e.g. equation 13 and equation 2 in Draine \& Lazarian 1998b and Dickinson et al. 2018, respectively), which suggests that a reduction in grain size from 6.3 to $6.0 \mathrm{~nm}$ would shift equipartition rotation frequencies $\tilde{v}$ from 30 to $31.5 \mathrm{GHz}$. The spectrum will be shifted accordingly, since for a Boltzmann distribution of rotation frequencies, the emergent emissivity $j_{v} / n_{\mathrm{H}}$ is modulated by a high frequency Boltzmann cut-off $\exp \left[-\frac{3}{2} v^{2} / \tilde{\nu}^{2}\right]$ (see equation 63 in Draine \& Lazarian 1998b).

Most of the SPDUST2 parameters were kept fixed in the optimization summarized in Table 4. Yet some of these parameters are expected to vary with depth into the PDR, and most particularly the radiation field. Variations in $\chi$ may also play a role in the spectral variations between the two SED extractions. We tested for the impact of such variations by optimizing a model for the SED in the $17 \mathrm{GHz}$ mask in which we decreased the UV field from our default value of $\chi=400$, to $\chi=100$. The result was a slightly better fit, with reduced $\chi_{r}^{2}=1.5$, and $a_{\text {cutoff }}=6.2 \pm 0.5 \AA$. Therefore, even with a $\times 4$ variation in the intensity of the UV field, an increasing $a_{\text {cutoff }}$ deeper into the PDR seems to be a robust prediction of the SPDUST2 models.

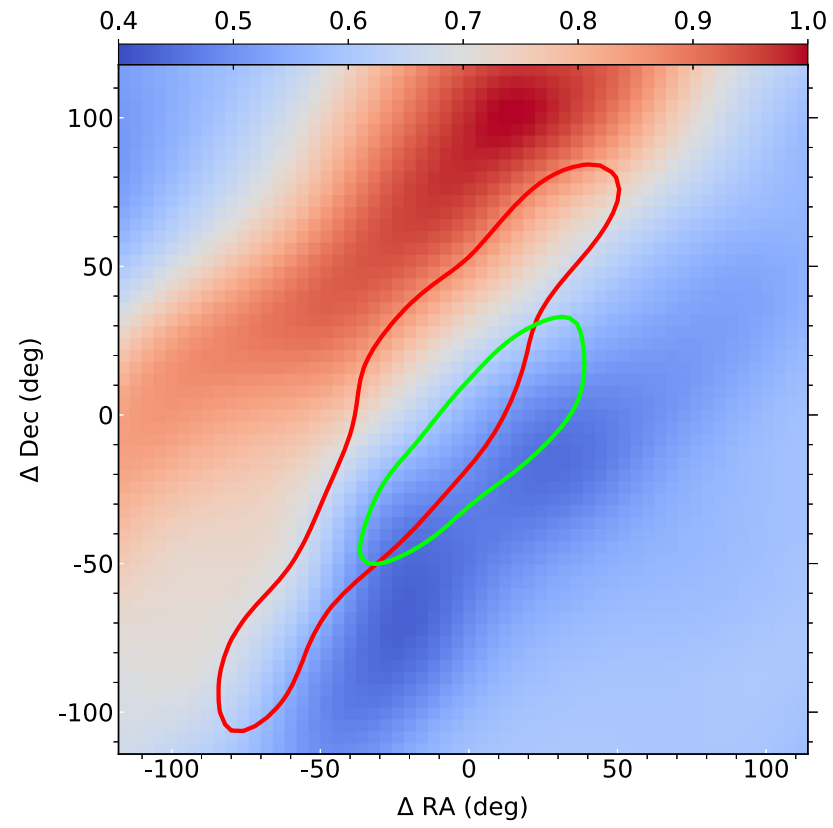

Figure 6. Comparison between the ATCA continuum and a proxy for PAH size. We show an overlay of a $17 \mathrm{GHz}$ contour (in red, at 85 per cent peak) and a $39 \mathrm{GHz}$ contour (in green, at 80 per cent peak), on the ratio of WISE 12 to $3.4 \mu \mathrm{m}$. The increasing value of the ratio deeper into the PDR is consistent with the larger PAHs.

Further support for an increasing PAH size deeper into the PDR can be found in a comparison with the WISE bands centred on 12 and $3.4 \mu \mathrm{m}$, which each correspond to PAH bands and whose ratio is a proxy for PAH size (Allamandola, Tielens \& Barker 1985; Ricca et al. 2012; Croiset et al. 2016). The relatively coarse angular resolution of the WISE images $(6.1 \mathrm{arcsec}$ at $3.4 \mu \mathrm{m}$ and $15 \mathrm{arcsec}$ at $12 \mu \mathrm{m})$, compared to IRAC $8 \mu \mathrm{m}(2.5 \mathrm{arcsec})$, prevents their filtering for the ATCA + SKYMEM response. But we can none the less degrade the WISE images to the coarsest ATCA beam (at $17 \mathrm{GHz}$ ) for a multifrequency comparison. The smoothed images are not exactly comparable to the ATCA mosaics, since we have not filtered for the ATCA response. But we hope that any resulting bias in the following analysis is small, since our synthesis imaging strategy corrects for missing ATCA antenna spacings using a prior image in SKYMEM, and the main source of point spread function sidelobes is due to fluxloss from missing antenna spacings at the centre of the $u v$-plane. We used the WISE images postprocessed as in (Arce-Tord et al. 2020) to produce Fig. 6 , which illustrates that the gradient in peak frequency across the filament is coincident with an increasing WISE $12 \mu \mathrm{m} / 3.4 \mu \mathrm{m}$ ratio. We quantify this trend using a standard Pearson correlation test $r$ (e.g. same as $r_{\text {sky }}$ in equation 11 of Arce-Tord et al. 2020), so similar to $r_{w}$ in equation (2) but without the weights, and instead adjusting the field of extraction to avoid the noise at the edge of the ATCA mosaics. The resulting Pearson $r$ are listed in Table 5. We recover the same trend as in Table 2, both $r$ and $r_{w}$ point at $20 \mathrm{GHz}$ as the best match to IRAC $8 \mu \mathrm{m}$. However, the ATCA map that best traces the shorter WISE wavelength is $39 \mathrm{GHz}$. The excellent correlation between ATCA $33 / 39 \mathrm{GHz}$ with $3.4 \mu \mathrm{m}$ and also between ATCA $20 / 17 \mathrm{GHz}$ with the $8 \mu \mathrm{m}$ template confirm the strong correlation between AME and PAH emission in this region.

The AME-PAH connection was put in doubt by Hensley, Draine \& Meisner (2016) based on a full-sky analysis on angular scales of 1 deg. Indeed, when taken as a whole, the $\rho$ Oph cloud is a 
Table 5. ATCA - WISE correlation statistics.

\begin{tabular}{lcc}
\hline $\begin{array}{l}\text { Frequency } \\
\text { (MHz) }\end{array}$ & WISE $3.4 \mu \mathrm{m}$ & IRAC $8 \mu \mathrm{m}$ \\
\hline 17481 & $0.01^{a}$ & 0.82 \\
20160 & 0.24 & 0.90 \\
33157 & 0.91 & 0.68 \\
39157 & 0.93 & 0.44 \\
\hline
\end{tabular}

${ }^{a}$ Pearson $r$ coefficients. All values bear a $1 \sigma$ uncertainty of 0.04 .

good example of the breakdown of the correlation between PAHs tracers and AME, since S 1, the brightest nebula in the complex in IRAC $8 \mu \mathrm{m}$ and also in Spitzer-IRS $11.3 \mu \mathrm{m}$ PAH band (Casassus et al. 2008, their table 2), has no detectable EME signal. However, it appears that EME and PAHs do correlate very tightly in higher angular resolution observations, and in regions where EME is present. Another example of excellent correspondence between AME and PAH emission is shown clearly in LDN1246 (Scaife et al. 2010).

The predictions for the grain-size distribution reported here should also be compared to the IR spectra available for $\rho$ Oph W. Under the spinning dust hypothesis for EME, a complete model should reproduce simultaneously the radio SED as well as the IR spectra, which are both due to the same underlying dust population. Here, we limit the scope of this report on the new ATCA observations to only the radio part, highlighting the need for a future modelling effort.

\section{CARBON RRL SEARCH}

The main spin-up mechanisms that could lead to VSG rotation frequencies of up to $\sim 30 \mathrm{GHz}$ may be either radiative torques or plasma drag (Draine \& Lazarian 1998b). Interestingly, the brightest nearIR nebulae in $\rho$ Oph, i.e. S 1 and SR 3, have no radio counterparts at centimetre wavelengths. Yet the circumstellar environments of embedded early-type stars correspond to the highest UV-radiation intensities. The absence of radio sources coincident with the IRbright circumstellar dust about S 1 and SR 3 cannot be reconciled with VSG depletion, as marginally shown by the CBI observations reported by Casassus et al. (2008), and confirmed with CBI2 in Arce-Tord et al. (2020). Radiative torques seem unlikely to explain the strong radio signal from the $\rho$ Oph W filament.

The alternative source of rotational excitation, plasma drag, is due to the interaction of the grain dipoles with passing ions - namely $\mathrm{H}^{+}$ or $\mathrm{C}^{+}$in the context of PDRs. If so the spinning dust emissivities would be best understood in terms of an emission measure: $I_{\mathrm{cm}} \propto$ $N_{\mathrm{VSG}} N\left(\mathrm{C}^{+}\right)$. An observational test of the plasma-drag hypothesis requires measurements of the $\mathrm{C}^{+}$abundance, as can be inferred using radio carbon recombination lines. The faint or absent centimetrewavelength signal from the circumstellar nebulae around S 1 and SR 3 in the radio maps may be due to these stars being too cold to create conspicuous C II regions (Casassus et al. 2008).

Pankonin \& Walmsley (1978) examined the most complete set of radio recombination line (RRL) data towards $\rho$ Oph to date. The line profiles observed at lower frequencies have widths of $1.5 \mathrm{~km} \mathrm{~s}^{-1}$ full width at half-maximum (FWHM). They mapped the neighbourhood of $\mathrm{S} 1$, but did not extend their coverage to $\rho$ Oph W, unfortunately. The highest frequency RRLs considered by Pankonin \& Walmsley (1978) are $\mathrm{C} 90 \alpha$ and $\mathrm{C} 91 \alpha$, at $\sim 9 \mathrm{GHz}$, which they interpreted as stemming from circumstellar gas about $\mathrm{S} 1$, with electron densities $n_{\mathrm{e}} \sim 15 \mathrm{~cm}^{-3}$ and $T_{\mathrm{e}} \sim 150 \mathrm{~K}$. This circumstellar $\mathrm{C}$ II region was inferred to be less than $\sim 2$ arcmin in diameter, and surrounded by a diffuse halo with $n_{\mathrm{e}} \sim 1 \mathrm{~cm}^{-3}$, traced by the lower frequency carbon RRLs.

We searched for carbon RRLs in the ATCA + CABB data, with a $2 \mathrm{GHz}$ bandwidth centred on $17481 \mathrm{MHz}$. Three $\alpha$-type RRLs fall into the $17481 \mathrm{GHz}$ IF: C71 $\alpha$ 18.00153, C72 $\alpha$ 17.26682, and C73 $\alpha$ 16.57156. No carbon RRLs are detected near the systemic velocity of $\rho$ OphW (which is $V_{\mathrm{lsr}}=+3 \mathrm{~km} \mathrm{~s}^{-1}$ Brown \& Knapp 1974; Pankonin \& Walmsley 1978). In the $17481 \mathrm{GHz}$ IF, the velocity width of each channel is $\sim 16 \mathrm{~km} \mathrm{~s}^{-1}$. The noise in single-channel reconstructions is $\sim 2 \mathrm{mJy}$ beam $^{-1}$, for a 30 arcsec beam FWHM. Assuming that the line is unresolved and is diluted in such broad channels, this upper limit is a factor of two looser than that obtained by Casassus et al. (2008) using Mopra.

For a rough estimate of carbon RRL intensities in $\rho \mathrm{Oph} \mathrm{W}$, we take a depth of $0.04 \mathrm{pc}$, which at a distance of $135 \mathrm{pc}$ subtends 1 arcmin, an ionization fraction of $10^{-4}$, due to carbon photoionization, $T_{\mathrm{e}}=$ $100 \mathrm{~K}$, and a $\mathrm{H}$-nucleus density of $n_{\mathrm{H}}=10^{5} \mathrm{~cm}^{-3}$ (these values are similar to those reported previously for $\rho$ Oph W, e.g. Habart et al. 2003). The peak intensity of the emergent $\mathrm{C} 71 \alpha$ is $18 \mathrm{mJy}_{\text {beam }}{ }^{-1}$, for local thermodynamic equilibrium, ${ }^{1}$ with a $1.5 \mathrm{~km} \mathrm{~s}^{-1}$ FWHM, and a 30 arcsec beam. When diluted in the $\sim 16 \mathrm{~km} \mathrm{~s}^{-1}$ channels of $\mathrm{CABB}$, the expected signal drops down to $\sim 1 \mathrm{mJy} \mathrm{beam}^{-1}$, or close to the limits obtained with Mopra. However, the expected CRRLs intensities should be within easy reach with the ALMA, as long as the spectral resolution is not degraded much beyond $\sim 0.5 \mathrm{~km} \mathrm{~s}^{-1}$. The spectra line data could be acquired as part of future observations to map the EME signal in $\rho \mathrm{Oph} \mathrm{W}$ at $\sim 40 \mathrm{GHz}$ with the Band 1 receivers currently under construction, and which should yield a noise level of $3 \mathrm{mJy}_{\text {beam }}^{-1}$ in $40 \mathrm{~min}$ and in $0.5 \mathrm{~km} \mathrm{~s}^{-1}$ channels.

\section{CONCLUSION}

ATCA + CABB multiconfiguration mosaics of the $\rho$ Oph W PDR resolve the filament with $\sim 30$ arcsec resolutions from 5 to $39 \mathrm{GHz}$. Since the signal fills the primary beam a special purpose imaging synthesis strategy (SKYMEM) was applied to compensate for fluxloss and mitigate sidelobe oscillations with the incorporation of an image prior.

The multifrequency $17-39 \mathrm{GHz}$ mosaics reveal spectral variations within $\rho$ Oph W. The radio signal follows the near-IR filament, but it is progressively shifted towards the UV source at higher frequencies. Such morphological differences in frequency reflect changes in the radio spectrum as a function of position in the sky. While the morphological trends with frequency are qualitative, the corresponding spectral variations in terms of the SEDs are not significant given the systematic uncertainties.

The SED of $\rho \mathrm{Oph} \mathrm{W}$, with a very narrow peak at $\sim 30 \mathrm{GHz}$, is reminiscent of spinning-dust. The physical conditions inferred under this hypothesis, using an optimization of selected free-parameters in the SPDUST package, are consistent with those derived in the literature, but require a minimum grain size cut-off and relatively large electric dipoles. The cut-off in the grain sizes is particularly well constrained as a standard ISM size distribution would shift the peak of the spectrum towards $\sim 90 \mathrm{GHz}$. The spinning dust model accounts for the measured intensities, and suggests that the qualitative morphological differences can be interpreted in terms of an increasing minimum grain size deeper into the PDR.

\footnotetext{
${ }^{1}$ The LTE deviations become important for $n=72$ at $T_{\mathrm{e}}<100 \mathrm{~K}$, i.e. the $n=$ 72 population departure coefficient relative to LTE is $b<1$ and the emergent intensities are proportionally fainter.
} 
Further sampling of the spinning dust spectrum in $\rho$ Oph W at $\sim 50 \mathrm{GHz}$ with ALMA, in the context of the data reported here, would provide strong constraints on the minimum PAH size. Eventually, the predictions obtained from the rotational emission of PAHs should be tested against a physical model for the IR PAH bands in $\rho$ Oph W.

\section{ACKNOWLEDGEMENTS}

We thank the referee, Yvette Chanel Perrott, who provided important input for the presentation of the SKYMEM algorithm and for the interpretation of the SED fits, in addition to constructive comments on the analysis and a thorough reading. We also acknowledge interesting discussions and comments from Kieran Cleary, Roberta Paladini, Jacques Le Bourlot, and Evelyne Roueff. SC acknowledges support from a Marie Curie International Incoming Fellowship (REA236176) and by FONDECYT grant 1171624. MV acknowledges support from FONDECYT through grant 11191205. GJW gratefully thanks the Leverhulme Trust for the award of an Emeritus Fellowship.

\section{DATA AVAILABILITY}

The SKYMEM package can be found at https://github.com/simonca sassus/SkyMEM. The corresponding author will provide help to researchers interested in porting SKYMEM to other applications. The SKYMEM code repository also includes, as an example application, the sky-plane version of the data underlying this article. The unprocessed visibility data set can be downloaded from the Australia Telescope Online Archive at https://atoa.atnf.csiro.au/. The corresponding author will share the calibrated visibility data on reasonable request.

\section{REFERENCES}

Ali-Haïmoud Y., 2014, MNRAS, 437, 2728

Ali-Haïmoud Y., Hirata C. M., Dickinson C., 2009, MNRAS, 395, 1055

Allamandola L. J., Tielens A. G. G. M., Barker J. R., 1985, ApJ, 290, L25

Arce-Tord C. et al., 2020, MNRAS, 495, 3482

Brown R. L., Knapp G. R., 1974, ApJ, 189, 253

Cárcamo M., Román P. E., Casassus S., Moral V., Rannou F. R., 2018, Astron. Comput., 22, 16

Casassus S., Cabrera G. F., Förster F., Pearson T. J., Readhead A. C. S., Dickinson C., 2006, ApJ, 639, 951

Casassus S. et al., 2008, MNRAS, 391, 1075

Casassus S. et al., 2018, MNRAS, 477, 5104

Casassus S. et al., 2019, MNRAS, 483, 3278

Castellanos P. et al., 2011, MNRAS, 411, 1137

Cepeda-Arroita R. et al., 2020, preprint (arXiv:2001.07159)

Cieza L. A. et al., 2019, MNRAS, 482, 698

Croiset B. A., Candian A., Berné O., Tielens A. G. G. M., 2016, A\&A, 590, A26

Davies R. D., Dickinson C., Banday A. J., Jaffe T. R., Górski K. M., Davis R. J., 2006, MNRAS, 370, 1125

Dickinson C. et al., 2018, New Astron. Rev., 80, 1

Draine B. T., Hensley B., 2012, ApJ, 757, 103

Draine B. T., Lazarian A., 1998a, ApJ, 494, L19+

Draine B. T., Lazarian A., 1998b, ApJ, 508, 157

Draine B. T., Lazarian A., 1999, ApJ, 512, 740

Elias J. H., 1978, ApJ, 224, 453

Finkbeiner D. P., Schlegel D. J., Frank C., Heiles C., 2002, ApJ, 566, 898

Gaia Collaboration, 2018, A\&A, 616, A1

Garufi A. et al., 2020, A\&A, 633, A82

Gold B. et al., 2011, ApJS, 192, 15

Habart E., Boulanger F., Verstraete L., Pineau des Forêts G., Falgarone E., Abergel A., 2003, A\&A, 397, 623

Hensley B. S., Draine B. T., 2017, ApJ, 836, 179

Hensley B. S., Draine B. T., Meisner A. M., 2016, ApJ, 827, 45

Hoang T., Lazarian A., 2016, ApJ, 821, 91

Hoang T., Draine B. T., Lazarian A., 2010, ApJ, 715, 1462
Hoang T., Vinh N.-A., Quynh Lan N., 2016, ApJ, 824, 18

Högbom J. A., 1974, A\&AS, 15, 417

Kogut A., Banday A. J., Bennett C. L., Gorski K. M., Hinshaw G., Reach W. T., 1996, ApJ, 460, 1

Lada C. J., Wilking B. A., 1984, ApJ, 287, 610

Le Petit F., Nehmé C., Le Bourlot J., Roueff E., 2006, ApJS, 164, 506

Leitch E. M., Readhead A. C. S., Pearson T. J., Myers S. T., 1997, ApJ, 486, $\mathrm{L} 23+$

Liseau R. et al., 1999, A\&A, 344, 342

Markwardt C. B., 2009, in Bohlender D. A., Durand D., Dowler P., eds, ASP Conf. Ser. Vol. 411, Astronomical Data Analysis Software and Systems XVIII. Astron. Soc. Pac., San Francisco, p. 251

Pankonin V., Walmsley C. M., 1978, A\&A, 64, 333

Pattle K. et al., 2015, MNRAS, 450, 1094

Pérez S., Casassus S., Baruteau C., Dong R., Hales A., Cieza L., 2019, AJ, 158,15

Planck Collaboration XX, 2011, A\&A, 536, A20

Planck Collaboration XII, 2013, A\&A, 557, A53

Planck Collaboration I, 2016a, A\&A, 594, A1

Planck Collaboration X, 2016b, A\&A, 594, A10

Ricca A., Bauschlicher Charles W. J., Boersma C., Tielens A. G. G. M., Allamandola L. J., 2012, ApJ, 754, 75

Ridge N. A. et al., 2006, AJ, 131, 2921

Sault R. J., Teuben P. J., Wright M. C. H., 1995, in Shaw R. A., Payne H. E., Hayes J. J. E., eds, ASP Conf. Ser. Vol. 77, Astronomical Data Analysis Software and Systems IV. Astron. Soc. Pac., San Francisco, p. 433

Scaife A. M. M. et al., 2009, MNRAS, 394, L46

Scaife A. M. M. et al., 2010, MNRAS, 403, L46

Silsbee K., Ali-Haïmoud Y., Hirata C. M., 2011, MNRAS, 411, 2750

Tibbs C. T. et al., 2012, ApJ, 754, 94

Vidal M. et al., 2011, MNRAS, 414, 2424

Vidal M., Dickinson C., Harper S. E., Casassus S., Witt A. N., 2020, MNRAS, 495,1122

Watson R. A., Rebolo R., Rubiño-Martín J. A., Hildebrandt S., Gutiérrez C. M., Fernández-Cerezo S., Hoyland R. J., Battistelli E. S., 2005, ApJ, 624, L89

Weingartner J. C., Draine B. T., 2001, ApJ, 548, 296

White G. J. et al., 2015, MNRAS, 447, 1996

Wilson W. E. et al., 2011, MNRAS, 416, 832

Ysard N., Verstraete L., 2010, A\&A, 509, A12

\section{APPENDIX A: IMAGE RECONSTRUCTION}

The traditional image-reconstruction algorithm Clean is not ideal for extended sources that fill the beam, especially with sparse $u v$ coverage. Initial trials at imaging using the MIRIAD task CLEAN resulted in large residuals, with an intensity amplitude much greater than that expected from thermal noise, and with a spatial structure reflecting the convolution of the negative synthetic sidelobes with the morphology of the source (see Fig. A1). Attempts to improve dynamic range using the MAXEN task in MIRIAD gave worse results. We therefore designed a special-purpose image reconstruction algorithm, based on sky-plane deconvolution (hereafter SKYMEM), and that allows the incorporation of priors to recover the larger angular scales.

The present sky-plane approach is an alternative to similar nonparametric imaging synthesis strategies based on a $u v$-plane approach, in which model visibilities are compared to the interferometer data. An example package for such $u v$-plane approaches is UVMEM (Casassus et al. 2006; Cárcamo et al. 2018), which has been applied to diffuse ISM data (such as the CBI and CBI2 observations of $\rho$ Oph, Casassus et al. 2008; Arce-Tord et al. 2020) as well as in compact sources (e.g. such as Very Large Array and ALMA observations of protoplanetary discs; Casassus et al. 2018, 2019; Pérez et al. 2019). Both the sky-plane and the $u v$-plane approaches should of course be equivalent, but in the sky plane, we avoid delicate issues with 

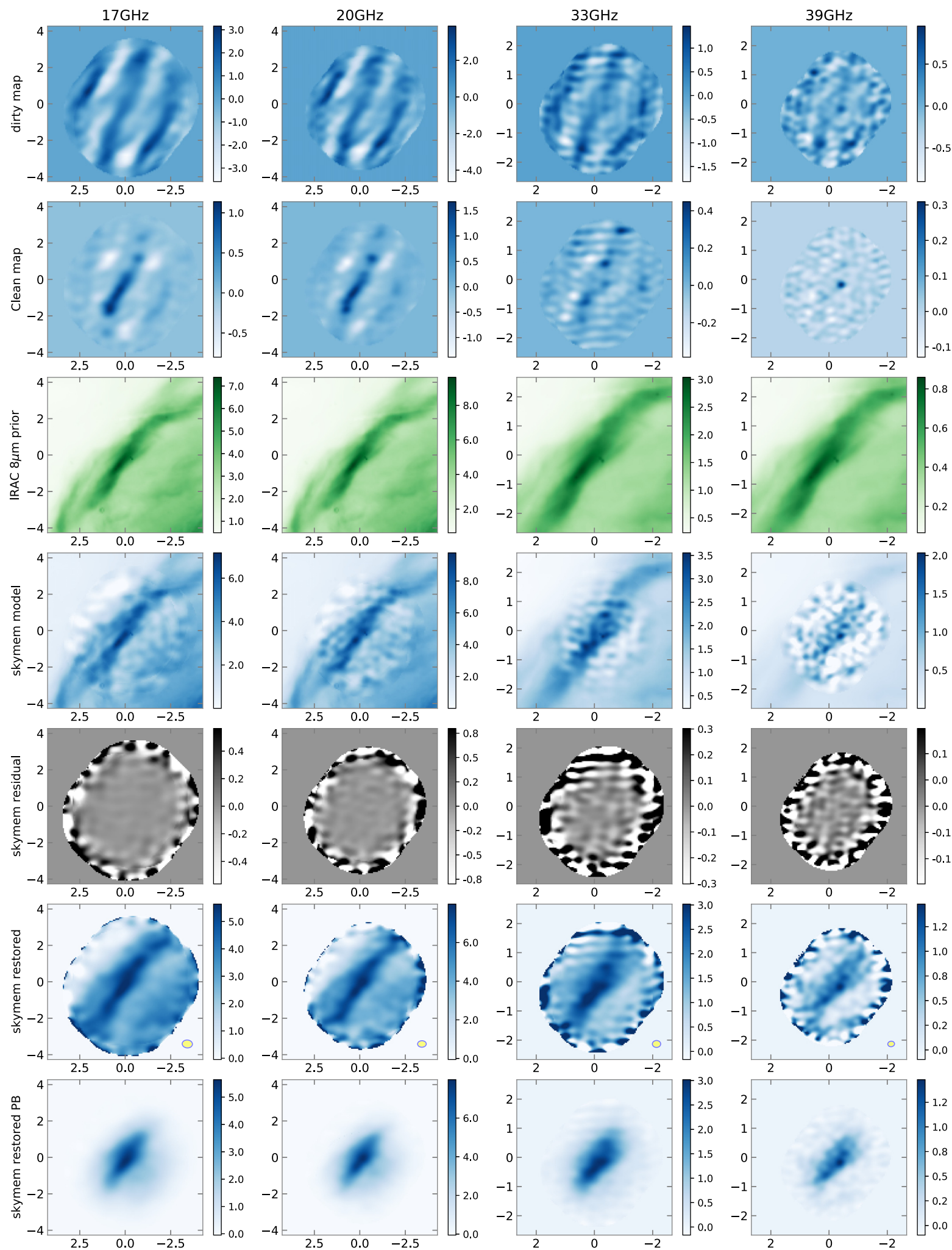

Figure A1. Synthesis imaging of the ATCA observations of $\rho$ Oph W. $x$ - and $y$-axis correspond to offset RA and Dec. in arcmin. This panel of images is organized as a table, where each image corresponds to the frequency given in column headers, for the synthesis imaging schemes given in line headers. We have, from top to bottom, the dirty and clean mosaics calculated with Miriad, the prior image scaled to each frequency, followed by the SKYMEM model image, its associated residual mosaic, and the restored mosaic, shown also after multiplication by the mosaic attenuation (labelled 'skymem restored PB'). The beam ellipses are shown in the restored images. The colour units are $\mathrm{mJy}_{\text {beam }}{ }^{-1}$. These images have not been point-source subtracted. 
Table A1. Scale factors for the SKYMEM priors.

\begin{tabular}{lcc}
\hline Frequency $^{a}$ & $\langle s\rangle^{b}$ & $\sigma(s)^{c}$ \\
\hline 5500 & 0.015 & - \\
8800 & 0.114 & - \\
17481 & 1.694 & 0.163 \\
20160 & 3.162 & 0.269 \\
33157 & 2.495 & 0.546 \\
39157 & 1.090 & 1.021 \\
\hline
\end{tabular}

Note. ${ }^{a}$ Centre frequency in MHz. ${ }^{b}$ Average and ${ }^{c}$ dispersion taken over all pointings.

Table A2. Dispersion of residuals and expected theoretical noise, in $\mu \mathrm{Jy}_{\text {beam }}{ }^{-1}$.

\begin{tabular}{lcc}
\hline Frequency $^{a}$ & $\sigma_{\circ}^{b}$ & $\sigma_{\text {ETC }}^{c}$ \\
\hline 5500 & 24 & 5 \\
8800 & 23 & 6 \\
17481 & 13 & 12 \\
20160 & 25 & 19 \\
33157 & 38 & 17 \\
39157 & 23 & 16 \\
\hline
\end{tabular}

Note. ${ }^{a}$ Centre frequency in MHz. ${ }^{b}$ Measured dispersion of SKYMEM residuals. ${ }^{c}$ Expected noise level in full scans, from the Exposure Time Calculator at https://www.narrabri.atnf.csiro.au/myatca/interactive_senscalc.html.

visibility gridding. In this application of SKYMEM, we rely entirely on the MIRIAD gridding machinery. ${ }^{2}$

In a sky-plane formulation of image synthesis, the data correspond to the dirty maps $\left\{I_{j}^{D}\right\}_{j=1}^{f}$ for each of the $f$ fields in the mosaic. In order to obtain a model sky image that fits the data, we need to solve the usual deconvolution problem, i.e. obtain the model image $I^{m}$ that minimizes a merit function $\mathcal{L}$ :

$\mathcal{L}=\chi^{2}-\lambda \mathcal{S}\left(I^{m}\right)$,

where

$\chi^{2}=\sum_{j=1}^{f} \sum_{i=1}^{n} w_{j}\left(\vec{x}_{i}\right)\left(I_{i}^{D}\left(\vec{x}_{i}\right)-I_{j}^{D m}\left(\vec{x}_{i}\right)\right)^{2}$.

The sums extend over the number of fields, $f$, and over the number of pixels in the model image, $n$. Each of the model dirty maps, $I_{j}^{D m}$, correspond to the convolution of the attenuated $I^{m}$ with the synthetic beam $B_{j}$,

$I_{j}^{D m}=\left(I_{j}^{m} A_{j}\right) * B_{j}$,

where $\left\{A_{j}\right\}_{j=1}^{f}$ are the primary beam attenuations for all fields. The weight map for a field $j$ is given by $w_{j}=1 / \bar{\sigma}_{j}^{2}$, where $\bar{\sigma}_{j}$ is the theoretical noise map as calculated with the 'sensitivity' option to the task INVERT in MIRIAD. This noise map is simply the thermal noise expected in the dirty map divided by the primary beam attenuation.

After several trials with a variety of functional forms for $\mathcal{S}$, we found that we obtained best results by simply using $\lambda=0$, i.e. with pure $\chi^{2}$ reconstructions. Image positivity of its own provided enough regularization. We used the Perl Data Language (PDL) for high-level data processing, and the optimization was carried out with a PDL-C patch to the Fletcher-Reeves algorithm in its GNU Scientific Library (GLS) implementation. We enforced positivity by clipping $I^{m}>0$

${ }^{2}$ The python version available on GITHUB is being integrated with the CASA framework. at each evaluation of $\mathcal{L}$ and its gradient. A couple of aspects of the implementation of SKYMEM are worth mentioning. In the convolution of equation (A3), the kernel should not be normalized, as would be the case for smoothing. Instead, to yield $I_{j}^{D m}$ in Jy beam ${ }^{-1}$ units, $B_{j}$ should be scaled by the number of pixels in a beam $\Omega_{G} /(\delta x)^{2}$, where $\Omega_{G}$ is the clean beam solid angle (see below) and $\delta x$ is the pixel scale. Another relevant aspect is the evaluation of the gradient of $\chi^{2}$, which can be written

$\frac{\partial \chi^{2}}{\partial I^{m}\left(\vec{x}_{i}\right)}=\sum_{j=1}^{f} \frac{2 A_{j}\left(\vec{x}_{i}\right)}{\bar{\sigma}^{2}}\left[\left(I^{D m}-I^{D}\right) \times B_{j}\right]_{\vec{x}_{i}}$.

The initial condition is important for the optimization as its parameter space is very structured. A blank initial image performed better than Clean, but yet lower values of $\mathcal{L}$ were obtained by starting with an image known to approximate the radio signal, which we call the prior image. The initial image we chose is the IRAC $8 \mu \mathrm{m}$ map, in its original angular resolution but filtered for point sources. This version of the IRAC $8 \mu \mathrm{m}$ map was multiplied by a representative dimensionless radio/IR correlation slope of $5.2 \times 10^{-4}$ (extrapolated from the slopes reported in Castellanos et al. 2011), so that the flux densities fall within the order of magnitude of the observed CBI flux densities in such sources. We then refined the intensity scale to exactly match that of the the ATCA observations in the following way. We simulated ATCA observation on the IRAC $8 \mu \mathrm{m}$ template, with identical $u v$-plane coverage as the observations, and calculated the dirty maps $\left\{I_{\text {IRAC } j}^{D}\right\}_{j=1}^{f}$ for each pointing $j$ in these mock data. The best-fitting correlation slopes $\left\{s_{j}\right\}_{j=1}^{f}$, defined by $I_{j}^{D}=s_{j} I_{\text {IRAC } j}^{D}$, are each given by

$s_{j}=\frac{\sum_{i} w_{j}\left(\vec{x}_{i}\right) I_{\mathrm{IRAC} j}^{D}\left(\vec{x}_{i}\right) I_{j}^{D}\left(\vec{x}_{i}\right)}{\sum_{i} w_{j}\left(\vec{x}_{i}\right)\left(I_{\mathrm{IRAC} j}^{D}\left(\vec{x}_{i}\right)\right)^{2}}$.

Finally, the prior image corresponds to this IRAC $8 \mu \mathrm{m}$ template scaled by $\langle s\rangle$, the mean correlation slope taken over all pointings. These prior images and their associated intensity scales are shown in Fig. A1. Table A1 lists the values for $\langle s\rangle$ and $\sigma(s)$ at each frequency. It is interesting to compare with the radio-IR correlation slopes $a(v)$ listed in Table 2. The larger dispersion of $s(v)$ with increasing frequency could reflect either real spectral changes. For the SKYMEM simulations on the IRAC template, all $s(v) \equiv 1$.

The resulting model images are shown in Fig. A1. It can be appreciated that the free parameters in the model image are modified relative to the input prior only within the field of the ATCA mosaic. It is also interesting to note that the low spatial frequencies of the prior are preserved, since the ATCA data provide no information that would constrain them.

Image restoration was obtained by smoothing the model image with the clean beam ${ }^{3} G$ in a reference field (that also sets the $\mathrm{Jy} \mathrm{beam}^{-1}$ units), and by adding the linear mosaic of dirty residuals $R^{D}$,

$I^{R}=I^{m} * G+R^{D}$.

The residual image for each pointing $j$ is $T_{j}^{D}=I_{j}^{D}-I_{j}^{D m}$, so

$R^{D}=\frac{\sum_{j} w_{j} T_{j}^{D} A_{j}}{\sum_{j} w_{j} A_{j}^{2}}$.

The residual and restored images are shown in Fig. A1. The residuals are adequately thermal, but the linear mosaic generated with the

\footnotetext{
${ }^{3}$ Which is an elliptical Gaussian.
} 
formula in equation (A7) amplifies the noise at the edges of the field. Thus we also provide in Fig. A1 a version of each restored images after multiplication by the mosaic attenuation pattern $\mathcal{A}$ to highlight the regions with smallest thermal errors, with

$\mathcal{A}(\vec{x})=\frac{\bar{\sigma}_{\circ}}{\bar{\sigma}_{R}(\vec{x})}$,

where $\bar{\sigma}_{\circ}$ is the minimum value in the theoretical noise image,

$\bar{\sigma}_{R}=\sqrt{\frac{1}{\sum_{j} w_{j} A_{j}^{2}}}$.

The dynamic range of the resulting SKYMEM images can be estimated by calculating the mean and dispersion of the residuals, i.e.

$\left\langle R^{D}\right\rangle=\frac{\sum_{j} \bar{w}_{R}\left(\vec{x}_{j}\right) R^{D}\left(\vec{x}_{j}\right)}{\sum_{j} \bar{w}_{R}\left(\vec{x}_{j}\right)}$, and

$\sigma_{\circ}=\sqrt{\frac{\sum_{j} \bar{w}_{R}\left(\vec{x}_{j}\right)\left(R^{D}\left(\vec{x}_{j}\right)-\left\langle R^{D}\right\rangle\right)^{2}}{\sum_{j} \bar{w}_{R}\left(\vec{x}_{j}\right)}}$.

The values for $\sigma_{\circ}$ are given in Table A2, where we see that they come close to the theoretical ATCA sensitivity. The noise image measured using the residuals rather than the theoretical sensitivy can be written as

$\sigma_{R}=\sigma_{\circ} \frac{\bar{\sigma}_{R}}{\bar{\sigma}_{\circ}}$.

This paper has been typeset from a $\mathrm{T}_{\mathrm{E}} \mathrm{X} / \mathrm{L} \mathrm{T}_{\mathrm{E}} \mathrm{X}$ file prepared by the author. 